\title{
Análisis y Comparación de la Información mostrada en las Webs Españolas y Extranjeras Relativa a Programas de Doctorado en el Área Agroforestal
}

\author{
Álvaro Sánchez-Medina ${ }^{(1)}$, Claudia García-Ventura ${ }^{(2)}$ y Esperanza Ayuga-Téllez ${ }^{(1) \star}$ \\ (1) Edificación, Infraestructuras y Proyectos para la Ingeniería Rural y Medioambiental (EIPIRMA), Univ. \\ Politécnica de Madrid, España. (alvaro.sanchezdemedina@upm.es; esperanza.ayuga@upm.es) \\ (2) Escuela Técnica Superior de Ingenieros de Montes, Forestal y del Medio Natural. Universidad \\ Politécnica de Madrid, España. (claudia.gventura@alumnos.upm.es)
}

* Autor a quien debe ser dirigida la correspondencia

Recibido Ene. 21, 2016; Aceptado Mar. 18, 2016; Versión final Abr. 18, 2016, Publicado Oct. 2016

\section{Resumen}

Se ha analizado la información sobre los programas de doctorado en el área de ingeniería agroforestal existente en las webs españolas y extranjeras. La visibilidad de la oferta formativa y la información de calidad son aspectos esenciales para fomentar el interés de los titulados universitarios a la hora de realizar sus estudios de doctorado. Esta información debería ser accesible y comparable entre instituciones, razón que motiva este estudio. Se han revisado 34 programas españoles y 33 de otros países (norteamericanos, europeos y asiáticos). Se han evaluado 43 ítems relacionados con la calidad de los recursos y los resultados del programa. Se ha incluido también, tres ítems relacionados con el formato y la estructuración de la web. La información más frecuente es: criterios de admisión, perfil de ingreso y número de plazas ofertadas, en todas las titulaciones. Los diseños de los sitios en general son poco atractivos, los contenidos escasos y el posicionamiento en buscadores de internet son mejorables en el $80 \%$ de los casos.

\section{Analysis and Comparison of the Information of Doctoral Programs shown in Spanish and Foreign Websites in the Area of Agroforestry}

\begin{abstract}
Current information of Spanish and foreign doctoral programs in websites of agroforestry engineering has been analyzed. The visibility of educational offer and quality information are essential issues to promote the interest of the university graduates to carry out their doctorate studies. This information should be accessible and comparable between institutions, reason that motivates this study. For this, 34 Spanish programs and 33 programs from other countries (American, European and Asian programs) have been checked. Forty three items related to the quality of the resources and the results of the program results have been evaluated. Also, three items related to the structure and design of the web sites were evaluated. The most common information in all degrees was: admission criteria, profile of candidates and the number of seats offered. The design of the web pages were generally unattractive, the contents were scarce, and the positioning in internet browsers could be improved in $80 \%$ of the cases.
\end{abstract}

Keywords: higher education; graduate; indications of quality; access network; contents 


\section{INTRODUCCIÓN}

Los estudios de posgrado son la cima de los procesos de formación. Preparan en la metodología y el desarrollo de nuevos conocimientos e innovaciones para la investigación (Cardoso, 2013). En España, los nuevos programas de doctorado siguen la normativa actual (Horgué, 2012) y deben tener en cuenta, además, los problemas detectados en programas anteriores (de Miguel, 2010). Los programas diseñados deben competir por el alumnado con una oferta de calidad que pueda ser verificada y comparable entre diferentes propuestas (Buela-Casal y Castro, 2009). La internacionalización de las universidades tiene gran importancia, especialmente en estudios de doctorado (Davila, 2012). El principal objetivo de la creación del Espacio Europeo de Educación Superior (EEES) es promover la integración europea y preparar la educación superior para afrontar los cambios a nivel mundial (Gijón Puertas y Moya, 2012). La formación doctoral tiene carácter internacional en sí misma. La Estrategia Universidad 2015 (Gobierno de España, 2008) está dirigida a la promoción de la excelencia, crear un sistema universitario internacional y adecuado a las demandas sociales. La universidad española se caracteriza por su modelo indisoluble entre la docencia y la investigación, proyectado en el ámbito del doctorado (Aznar et al, 2014).

Aunque con frecuencia se confunden y están relacionadas la internalización y la globalización tienen diferentes expresiones en las enseñanzas de doctorado (Altbach y Knight, 2007). Los resultados de la globalización incluyen la integración de la investigación, el uso de una lengua franca para la comunicación científica, un mercado internacional del trabajo científico, etc. La globalización facilita la comunicación y difusión de conocimientos y ha intensificado la competencia, como lo demuestra el número creciente de rankings nacionales e internacionales de clasificación (Krüger y Molas, 2010) y la atención prestada a los mismos (González-Riaño et al., 2014). Según el estudio de la European University Association (EUA) la competitividad es un aspecto relevante para el $38 \%$ de las instituciones siendo el más importante para el $60 \%$ de las instituciones la mejora de la calidad (Sursock y Smidt, 2010). Para fomentar la internalización y globalización de los programas de doctorado se deben emplear nuevas tecnologías como tele-conferencias o e-learning y aumentar las oportunidades de los doctorandos para que su trabajo se difunda internacionalmente, por ejemplo, mediante un incremento de becas de movilidad internacional, organización de talleres, conferencias y escuelas de verano, desarrollo de más programas de doctorado conjuntos y la codirección de trabajos (Ayuga-Téllez, 2010).

En general, el número de estudiantes en titulaciones de tercer ciclo ha variado en cada país de forma diferente. En el periodo 2009-2012, los países europeos han experimentado un aumento mayor que en otros continentes (Eurostat, 2014), por ejemplo en Países Bajos (22,1\%), Bélgica (11\%), Suecia (6,8\%), España $(8,4 \%)$ y Francia (5\%). En Estados Unidos la cifra se incrementó en un $9 \%$. En cuanto a las titulaciones asiáticas, solo se han obtenido estadísticas sobre Japón en el cual el aumento es próximo a cero. La evolución en 2009-2012 del número de estudiantes de tercer ciclo del conjunto de la Unión Europea (Eurostat, 2014) muestra resultados más positivos sobre las cifras españolas que los de la Europa de los 28. Mientras España registra un aumento del 8,4\%, el conjunto de los países se queda en un 3,1\%. En España se regulan las enseñanzas de doctorado en 2011 integrándose al EEES y al Espacio Europeo de Investigación (EEI). Coincidiendo con esta reforma, el número de estudiantes españoles ha ido decayendo desde el curso 2011-12 hasta el 2014-15 en un 5,83\% con una tasa media del 1,95\% (MECD, 2015).

Es pronto para poder relacionar esta disminución de estudiantes con una causa concreta pero, por encima de circunstancias que escapan a los gestores universitarios, es importante ser conscientes del problema y tomar decisiones que atraigan titulados universitarios a los estudios de doctorado, mejorando los programas, aumentando su internacionalización y haciendo más visibles los resultados, la calidad y la eficiencia (Benito y Romera, 2012). También sería necesario mejorar la calidad de los medios que difunden la oferta de programas de posgrado y proporcionar datos fácilmente accesibles, que permitan conocer en profundidad las características, medios o recursos disponibles y resultados visibles de formación de dichos programas. A la expansión de la oferta y demanda de programas de posgrados se asocian altas tasas de deserción y, al intento de evaluarlas, ausencia de información válida y de confianza (Wainerman y Tuñón, 2013). En el área de la ciencia y la ingeniería el número de doctores en los últimos años en Europa se ha mantenido, e incluso ha disminuido (Moguerou, 2005). Desde esta perspectiva, resulta de gran interés ofrecer información de calidad que pueda compararse con la de otras instituciones (Fornas Carrasco, 2003) para facilitar a los interesados la elección de programa y universidad (Aguado et al., 2011). Para ello es indispensable recurrir a los medios actuales de comunicación y evaluar las fuentes de información publicadas en Internet (Ayuso García y Martínez Navarro, 2005).

Una página web es, en esencia, una tarjeta de presentación digital, ya sea para empresas, organizaciones, personas, etc. Así mismo, la nueva tendencia orienta a que las páginas web no sean sólo atractivas para los internautas, sino también optimizadas para que sean rápidamente localizadas por buscadores a través del código fuente. Conseguir este doble propósito puede crear conflictos respecto de la calidad del contenido. 
Actualmente las Universidades están haciendo un esfuerzo por mejorar la calidad de sus páginas web (AlDebei, 2014, Pinto et al., 2014). El objetivo del presente trabajo es analizar la información relativa a los programas de doctorado del área de ingeniería agroforestal incluida en sus páginas web y evaluar éstas desde el punto de vista de su atractivo y facilidad de uso.

\section{MATERIALES Y MÉTODOS}

\section{Selección de las Titulaciones}

Las titulaciones de las universidades Españolas se han seleccionado a partir del trabajo realizado por el Ministerio de Educación (Ayuga-Téllez, 2010). Así, se han considerado las titulaciones de las universidades mejor situadas en el ranking Webometrics, basado en la información y uso de las webs universitarias (Webometrics, 2015), y relacionadas directamente con el área agroforestal. Todas ellas pertenecen a Universidades públicas, de tal modo que se analizarán 34 titulaciones correspondientes a 23 universidades (tabla 1). Las titulaciones extranjeras seleccionadas universidades que oferten programas de doctorado relacionados directamente con el área agroforestal. El criterio básico de selección ha sido su participación activa en las redes temáticas europeas ERABEE y USAEE y también que su posición en el ranking mundial Webometrics sea por encima del tercio superior con respecto al total. De este modo se han seleccionado 33 titulaciones del área agroforestal correspondientes a 18 universidades (tabla 1), todas públicas excepto la Universidad de Kyoto (Japón). Hay 6 universidades europeas (6 titulaciones), 8 universidades norteamericanas (15 titulaciones) y 4 universidades asiáticas (12 titulaciones).

Tabla 1. Titulaciones extranjeras seleccionadas

\begin{tabular}{|c|c|c|}
\hline Título & Universidad & País \\
\hline Agricultural sciences of food and environment & AgroSup Dijon & Francia \\
\hline Science, Engineering \& Technology & Ku Leuven & Bélgica \\
\hline Climate \& Water & Wageningen & Holanda \\
\hline Biodiversita', agricoltura e ambiente & Università degli studi di Bari Aldo Moro & Italia \\
\hline Sustainable use of renewable natural resources & University of Helsinki & Suecia \\
\hline Resources Management and agricultural engineering & Agricultural University of Athens & Grecia \\
\hline Biological Systems Engineering & Virginia Tech & \multirow[t]{13}{*}{ EE.UU } \\
\hline Agricultural and Resource Economics & University of California & \\
\hline Applied Plant Sciences Graduate Program & University of Minessota & \\
\hline Agricultural and Applied Economics & \multirow[t]{4}{*}{ University of Illinois } & \\
\hline Agricultural and Biological Engineering & & \\
\hline Crop Sciences & & \\
\hline Natural Resources and Environmental Sciences & & \\
\hline Animal Science & \multirow[t]{5}{*}{ Cornell University } & \\
\hline Biological and Environmental Engineering & & \\
\hline Food Science and Technology & & \\
\hline Natural Resources & & \\
\hline Plant Breeding & & \\
\hline Environmental \& Forest Sciences & University of Washington & \\
\hline Forest conservation biology and wildlife ecology & University of Toronto. Faculty of Forestry & \multirow[t]{2}{*}{ Canadá } \\
\hline Forest biology \& management & $\begin{array}{l}\text { University of Alberta. Faculty of Agricultural, life } \\
\text { \& environmental science }\end{array}$ & \\
\hline Agriculture & University of Kyoto & Japón \\
\hline Agronomy & \multirow[t]{7}{*}{ National Taiwan University } & \multirow[t]{7}{*}{ Taiwán } \\
\hline Agriculture & & \\
\hline Forestry & & \\
\hline Animal science and technology & & \\
\hline Agricultural Economics & & \\
\hline Horticulture & & \\
\hline Food Science and Technology & & \\
\hline Water and environmental engineering & \multirow[t]{3}{*}{ University of Hong-Kong } & \multirow[t]{4}{*}{ China } \\
\hline Ecology and biodiversity & & \\
\hline Food and nutritional science & & \\
\hline Biology & The Chinese University of Hong-Kong & \\
\hline
\end{tabular}


Análisis de contenidos relativos a la información recogida en la web.

La metodología empleada para el análisis de la información fue el Análisis de Contenidos que se centra en la descripción de contenidos (método para estudiar y analizar las comunicaciones de una forma sistemática, objetiva y cuantitativa y la metodología comparada (Noguero, 2002). Así, se accedió a todas y cada una de las páginas webs de los programas de doctorado considerados y se efectuó el análisis de contenido recomendado por Aguado et al. (2011), que incluye 35 ítems, añadiendo otros aspectos evaluados por las agencias de acreditación españolas. En total se analizaron 43 ítems, registrando la existencia (SI) o no (NO) de información en cada uno de los ítems, para cada una de las titulaciones.

La lista de contenidos, agrupados por temas, fue la siguiente:

(1) Accesibilidad: plazas ofertadas, alumnos de nuevo ingreso, alumnos matriculados, criterios de admisión, perfil de ingreso y coste de la matrícula;

(2) Medios utilizados: si existe información sobre los medios técnicos disponibles, sobre el profesorado, su número y si hay información sobre ellos, más allá de su nombre y correo electrónico;

(3) Programa: complementos de formación ofertados, actividades obligatorias para los alumnos, actividades optativas, ECTS a cursar e idiomas en los que se puede realizar el trabajo de tesis;

(4) Resultados de la difusión de la actividad investigadora: publicaciones relacionadas con las tesis desarrolladas, trabajos en revistas internacionales o nacionales con factor de impacto (FI), publicaciones en otras revistas internacionales y nacionales, comunicaciones presentadas en congresos, informes, capítulos de libros y libros completos;

(5) Relevancia de los resultados de la investigación (R.I.): proyectos de investigación relacionados con las tesis doctorales y el número de alumnos becados;

(6) Eficacia de los resultados (E.R.): tesis rechazadas en primera vuelta para su corrección, tesis terminadas, calificaciones de las tesis terminadas y tiempo medio de realización de las tesis;

(7) Internacionalización de los programas (INTERNAC.): existencia de conferenciantes extranjeros, evaluadores externos a la Universidad, universidades participantes (españolas y extranjeras), estancia en centros de investigación extranjeros durante el desarrollo de las tesis doctorales;

(8) Calidad de los programas: menciones de Doctor Europeo o similares, menciones de excelencia al programa, Premios Extraordinarios de Doctorado y de Tesis Doctorales, existencia de mención de calidad, existencia de Sistema Interno de Garantía de la Calidad (SIGC) y existencia de buzón de sugerencias, realización de encuestas de satisfacción a ingresados y a egresados, existencia de datos sobre la orientación profesional del programa, porcentaje de doctores egresados con empleo y porcentaje de doctores egresados con trabajo en investigación y docencia.

\section{Análisis de contenidos relativos al diseño, estructuración y accesibilidad de la web.}

Existen tres aspectos de las webs muy importantes cuando se plantea la difusión de los programas de doctorado (Ayuga-Téllez, 2010), que son: (1) Diseño: se ha valorado este aspecto sobre la información ofrecida con la siguiente puntuación: 1 = "muy poco atractiva"; 2 = "poco atractiva"; 3 = "atractiva"; 4 = "muy atractiva"; (2) Estructuración: se ha valorado este aspecto en relación a la información ofrecida con la siguiente puntuación: 1 = "muy mal estructurada"; 2 = "mal estructurada"; 3 = "bien estructurada"; 4 = "muy bien estructurada; y (3) Accesibilidad: se puntúa de 1 a 4 según el posicionamiento en el buscador Google, con el indicador de búsqueda en castellano "doctorado máster agrícola forestal" y en inglés " forestry agriculture master doctorate". Se emplea la siguiente puntuación: $1=$ por encima del puesto 15ㅜ; 2 = entre el puesto $11^{\circ}$ y $15^{\circ} ; 3=$ entre el puesto $6^{\circ}$ y $10^{\circ} ; 4=$ entre el puesto 10 y $5^{\circ}$. Tanto el diseño como la estructura fueron evaluados por una estudiante de doctorado. La accesibilidad fue evaluada por los investigadores.

\section{Análisis estadístico}

Se calculó el porcentaje de programas de doctorado en cuyas páginas web aparece información sobre el ítem analizado. La existencia de esta información en la web se ha diferenciado en 8 conjuntos temáticos. Además, para los ítems de los 8 conjuntos se ha realizado un test de diferencia de proporciones entre los siguientes grupos: España, Europa, América, Asia y Total Extranjero (todos los países menos España) y se han calculado los p-valores de los ítems que presentan diferencias estadísticamente significativas (al 95\% de confianza) entre España y el resto de dichos grupos. La valoración relativa al diseño, estructuración y accesibilidad se ha analizado según el porcentaje de webs que alcanzan las puntuaciones consideradas.

\section{RESULTADOS Y DISCUSIÓN}

En primer lugar se exponen los resultados, en porcentaje, de la existencia en las páginas web de los contenidos analizados por grupos temáticos (tabla 2). 
Tabla 2.Porcentaje de programas, nacionales y extranjeros, que presentan en su web los ítem analizados.

\begin{tabular}{|c|c|c|c|c|c|c|}
\hline & & España & Europa & América & Asia & $\begin{array}{c}\text { Total } \\
\text { Extranjero }\end{array}$ \\
\hline \multirow{6}{*}{ 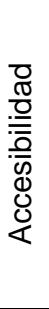 } & Plazas ofertadas & 67,6 & 28,6 & 6,7 & 8,3 & 8,8 \\
\hline & Alumnos matriculados & 5,9 & 14,3 & 13,3 & 8,3 & 11,8 \\
\hline & Alumnos nuevo ingreso & 5,9 & 0,0 & 6,7 & 0,0 & 2,9 \\
\hline & Criterios de admisión & 91,2 & 71,4 & 86,7 & 41,7 & 67,6 \\
\hline & Perfil de ingreso & 82,4 & 57,1 & 66,7 & 8,3 & 44,1 \\
\hline & Coste de la matricula & 8,8 & 42,9 & 40,0 & 25,0 & 35,3 \\
\hline \multirow{3}{*}{$\begin{array}{l}\text { ஜ } \\
\stackrel{0}{0} \\
\stackrel{\Phi}{\Sigma}\end{array}$} & № profesores & 64,7 & 28,6 & 13,3 & 8,3 & 14,7 \\
\hline & Información profesorado & 26,5 & 28,6 & 20,0 & 0,0 & 14,7 \\
\hline & Información medios técnicos disponibles & 41,2 & 14,3 & 0,0 & 58,3 & 23,5 \\
\hline \multirow{5}{*}{ 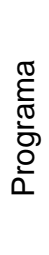 } & Complementos de formación ofertados & 26,5 & 14,3 & 6,7 & 0,0 & 5,9 \\
\hline & Actividades obligatorias & 55,9 & 14,3 & 20,0 & 41,7 & 26,5 \\
\hline & Actividades optativas & 41,2 & 14,3 & 0,0 & 0,0 & 2,9 \\
\hline & ECTS a cursar & 47,1 & 28,6 & 20,0 & 66,7 & 38,2 \\
\hline & Idiomas en que se puede realizar la tesis & 5,9 & 42,9 & 0,0 & 0,0 & 8,8 \\
\hline \multirow{7}{*}{ 㖣 } & Publicaciones internacional o nacional FI & 23,5 & 14,3 & 6,7 & 0,0 & 5,9 \\
\hline & Publicaciones internacionales (otras) & 0,0 & 14,3 & 6,7 & 0,0 & 5,9 \\
\hline & Publicaciones nacionales (otras) & 5,9 & 14,3 & 6,7 & 0,0 & 5,9 \\
\hline & Comunicaciones Congresos & 2,9 & 0,0 & 6,7 & 0,0 & 2,9 \\
\hline & Informes & 0,0 & 0,0 & 0,0 & 0,0 & 0,0 \\
\hline & Capítulos libros & 0,0 & 0,0 & 6,7 & 0,0 & 2,9 \\
\hline & Libros & 0,0 & 0,0 & 6,7 & 0,0 & 2,9 \\
\hline \multirow{2}{*}{$\dot{\dot{q}}$} & Proyectos investigación & 14,7 & 28,6 & 6,7 & 0,0 & 8,8 \\
\hline & Alumnos becados & 0,0 & 0,0 & 0,0 & 0,0 & 0,0 \\
\hline \multirow{4}{*}{ 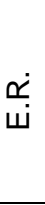 } & Tesis rechazadas $1^{\circ}$ vuelta para corrección & 0,0 & 0,0 & 0,0 & 0,0 & 0,0 \\
\hline & Tesis terminadas & 35,3 & 14,3 & 0,0 & 0,0 & 2,9 \\
\hline & Calificaciones de las tesis terminadas & 20,6 & 0,0 & 0,0 & 0,0 & 0,0 \\
\hline & Tiempo medio realización tesis & 2,9 & 0,0 & 0,0 & 0,0 & 0,0 \\
\hline \multirow{5}{*}{ 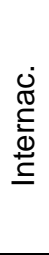 } & Conferencias extranjeros & 0,0 & 0,0 & 0,0 & 0,0 & 0,0 \\
\hline & Univ. participantes españolas & 26,5 & 14,3 & 0,0 & 0,0 & 2,9 \\
\hline & Univ. participantes extranjeras & 20,6 & 0,0 & 0,0 & 0,0 & 0,0 \\
\hline & Estancia Centros inv. extranjero & 2,9 & 0,0 & 0,0 & 0,0 & 0,0 \\
\hline & Evaluadores externos a la Universidad & 0,0 & 0,0 & 0,0 & 0,0 & 0,0 \\
\hline \multirow{11}{*}{ 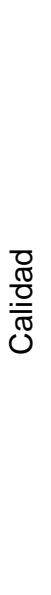 } & Mención excelencia & 26,5 & 14,3 & 0,0 & 0,0 & 2,9 \\
\hline & Mención de calidad & 5,9 & 0,0 & 0,0 & 0,0 & 0,0 \\
\hline & Sistema Interno de Garantía de Calidad (SIGC) & 61,8 & 0,0 & 0,0 & 0,0 & 0,0 \\
\hline & Realización encuestas a ingresados & 0,0 & 14,3 & 0,0 & 0,0 & 2,9 \\
\hline & Realización encuestas a egresados & 0,0 & 0,0 & 0,0 & 0,0 & 0,0 \\
\hline & Buzón de sugerencias & 38,2 & 14,3 & 0,0 & 0,0 & 2,9 \\
\hline & Orientación profesional & 11,8 & 28,6 & 0,0 & 0,0 & 5,9 \\
\hline & Premios Ext. Doctorado & 0,0 & 0,0 & 0,0 & 0,0 & 0,0 \\
\hline & Premios Tesis Doctorales & 0,0 & 0,0 & 0,0 & 0,0 & 0,0 \\
\hline & $\%$ doctores egresados con trabajo & 0,0 & 0,0 & 0,0 & 0,0 & 0,0 \\
\hline & $\begin{array}{l}\text { \% doctores egresados trabajo en investigación y } \\
\text { docencia }\end{array}$ & 5,9 & 0,0 & 0,0 & 0,0 & 0,0 \\
\hline
\end{tabular}

La presencia de los ítems analizados es más común en el caso de los tres primeros conjuntos temáticos: accesibilidad, medios utilizados y programa, que son los más importantes para el futuro estudiante, en 
cuanto a la información básica de los programas. En el resto, la presencia de ítems es reducida o nula en alguno de los continentes. Si analizamos la presencia general de los ítems por grupos de continentes, destacan América y Asia en los que están ausentes 25 y 34 de los 43 analizados. En las titulaciones españolas, el número de ítems presentes es similar a los de las europeas. Los ítems ausentes en todos los continentes son: Informes, alumnos becados, tesis rechazadas en primera vuelta, evaluadores externos, premios extraordinarios de doctorado y de tesis doctoral y doctorados egresados con trabajo no relacionado con la investigación y docencia; algunos de ellos son indicadores comunes de la calidad de las tesis realizadas, así como de la importancia de los grupos de investigación asociados al programa de doctorado.

Con el objetivo de conocer si existen diferencias estadísticamente significativas entre la información disponible en las páginas web de los programas se aplicó el test de diferencia de proporciones. Se analizó la diferencia entre la información disponible sobre los programas españoles y los de cada continente; también se comparó entre los programas españoles y el total de los programas extranjeros (Total Extranjero). De forma global, las mayores diferencias se presentan cuando se comparan las titulaciones españolas con las no españolas (tabla 3), obteniendo diferencias en 14 ítems; respecto a las asiáticas se reduce a 10, con las americanas se diferencia en 9 , y con las europeas es donde se presentan más similitudes, con sólo 6 ítems con diferencias estadísticamente significativas. Si se analiza cada conjunto temático, se encuentran diferencias en todos a excepción del relativo a los resultados de investigación.

Tabla 3. P-valores del test de diferencia de proporciones aplicado a los programas por continentes (sólo se muestran los valores de las variables con diferencias estadísticamente significativas al 95\%).

\begin{tabular}{|l|c|c|c|c|}
\hline & Europa & América & Asia & Total Extranjero \\
\hline Plazas ofertadas & & 0 & 0,0004 & 0 \\
\hline Criterios de admisión & & & 0,0003 & 0,0161 \\
\hline Perfil de ingreso & & & 0 & 0,0011 \\
\hline Coste de la matricula & 0,0201 & 0,0093 & & 0,0084 \\
\hline № profesores & & 0,0009 & 0,0008 & 0,0028 \\
\hline Información profesorado & & 0,0467 & \\
\hline Información sobre medios técnicos disponibles & & 0,0033 & & \\
\hline Complementos de formación ofertados & & & 0,0273 & 0,0212 \\
\hline Actividades obligatorias & 0,0449 & 0,0199 & & 0,0138 \\
\hline Actividades optativas & & 0,0033 & 0,0077 & 0,0001 \\
\hline Idiomas en que se puede realizar la tesis & 0,0065 & & & \\
\hline Publicaciones internacional o nacional FI & & & & 0,0404 \\
\hline Publicaciones internacionales (otras) & 0,0241 & & & \\
\hline Tesis terminadas & & 0,0081 & 0,0167 & 0,0007 \\
\hline Calificaciones de las tesis terminadas & & & & 0,0052 \\
\hline Univ. participantes españolas & & 0,0273 & 0,0467 & 0,006 \\
\hline Univ. participantes extranjeras & & & & 0,0052 \\
\hline Mención excelencia & & 0,0273 & 0,0467 & 0,006 \\
\hline Sistema de garantía de calidad (SIGC) & 0,0029 & 0,0001 & 0,0002 & 0 \\
\hline Realización encuestas a ingresados & 0,0256 & & & \\
\hline Buzón de sugerencias & & & & 0,0003 \\
\hline
\end{tabular}

En relación a la accesibilidad del programa, el ítem con mayores diferencias entre titulaciones españolas y el resto, es el del coste de la matrícula. En España se ofrece menos información $(8,8 \%$ frente a $40 \%$ de media para el resto). Los precios públicos medios del crédito matriculado en España aumentó en el periodo 2011-2014 con una tasa anual del 2,9\% (MECD, 2015). Por ello resulta destacable que no se refleje esa información, dada la situación económica general del país. Otros ítems relevantes para la toma de decisiones del estudiante como criterios de admisión o perfil de ingreso, difieren menos entre continentes. En cuanto a los medios humanos y técnicos disponibles, las mayores diferencias se encuentran en la información del número de profesores adscritos al programa, en concreto con los programas americanos y asiáticos. La información detallada del profesorado, como se ha comprobado en la tabla 1, es escasa, y solo aparece una ligera diferencia con los títulos asiáticos La no inclusión de esta información supone al alumno el desconocimiento de las líneas de investigación disponibles o de los investigadores que las desarrollan. 
En relación al programa en sí, el ítem con mayor diferencia entre programas españoles y el resto es el de las actividades optativas; en concreto se obtienen $\mathrm{p}$-valores muy bajos al comparar con programas americanos $(0,0033)$, asiáticos $(0,0077)$ y con los extranjeros en general $(0,0001)$. La información sobre los idiomas en los que se puede realizar la tesis es escasa, encontrando diferencias entre los programas españoles y los europeos ( $p$-valor $=0,0065)$, siendo este dato más habitual en los segundos $(42,9 \%$ ) que en los primeros (5,9\%). El resultado es destacable, dado que en España la lengua oficial es el castellano, pero también existen lenguas cooficiales como el catalán, gallego y vasco. Por otro lado, cada vez es más frecuente que los futuros doctores opten por la modalidad de Doctorado Internacional, y por la cual varios apartados del trabajo final deben estar en dos idiomas (Horgué Baena, 2012).

Los resultados para los ítems relativos a la difusión de la actividad investigadora muestran leves diferencias entre España y el resto en cuanto a las publicaciones internacionales o nacionales con FI; también existen diferencias, aunque menores, con los programas americanos. En el caso de la evaluación de los resultados del programa y su eficacia y la Internacionalización de los Programas los resultados del test son similares. En el caso de la evaluación de resultados, el ítem con mayores diferencias es el de número de tesis terminadas comparando los programas españoles con los demás. La calificación de dichas tesis es una información casi inexistente. En cuanto a la internacionalización de los programas, la diferencia más destacable es la de los programas españoles con los demás programas referente a las universidades participantes tanto nacionales como extranjeras.

En cuanto a los ítems relativos a la calidad del programa, también se observan diferencias significativas, como por ejemplo en la existencia de información sobre el SIGC, entre los programas españoles y los europeos, americanos y asiáticos, siendo en estos dos últimos continentes inexistente. También aparecen diferencias significativas en el ítem sobre encuestas a ingresados, siendo la información sobre estas encuestas más frecuente en los programas europeos que españoles. Sobre orientación profesional, tanto en programas españoles como europeos, no se presenta información sobre los egresados y tampoco sobre la realización de encuestas de satisfacción a este colectivo, siendo este último ítem relevante en la elección de un programa por parte de los alumnos (Hernandez et al., 2012).

El diseño es uno de los primeros aspectos que invita al usuario a permanecer en la página web. Este es el factor más valorado por los internautas a la hora de decidir sobre la elección de un servicio a través de la web. Un bonito diseño da sensación de seguridad (Robins y Holmes, 2008) ya que parece que la web ha sido más trabajada también en otros aspectos (seguridad, uso de nuevas técnicas, etc). Los resultados del análisis del diseño (Figura 1) revelan que el $81 \%$ de de los programas españoles tienen webs con diseño poco o muy poco atractivo y tan sólo un 19\% tienen una web atractiva o muy atractiva, lo que refleja que es un aspecto poco cuidado a la hora de difundir la información. En las web de otros países, el $65 \%$ se han clasificado como atractivas y el $35 \%$ como poco atractivas. La mayoría son páginas web con un diseño muy elaborado (mayor variedad de colores, fotografías dinámicas, formato pantalla completa) que invitan al usuario a seguir navegando por la web.

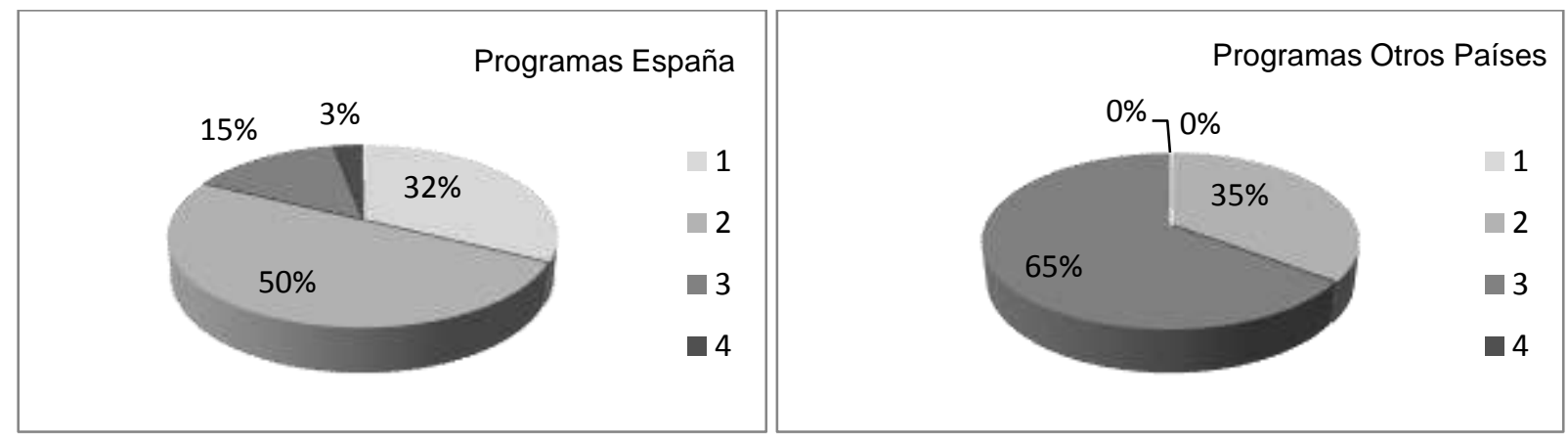

Fig. 1. Porcentaje de webs según el atractivo de su diseño (1 = "muy poco atractiva", 2 = "poco atractiva" $3=$ "atractiva" y 4 = "muy atractiva")

Por estructuración se entiende una estructura de la información adecuada y facilidad de uso. Es importante que el usuario encuentre un sitio web que satisfaga sus necesidades. Es necesario que el diseño se centre en los usuarios, para que puedan interactuar con los sitios de la forma más fácil, cómoda e intuitiva. En este caso, se debe suponer que el usuario es el titulado superior que busca información para decidir si continúa su formación como investigador, o bien, si está decidido, seleccionar el programa más apropiado para ello. Por la internacionalidad de los programas hay pensar en un posible candidato de cualquier parte del mundo. En la figura 2 se observa cómo el $53 \%$ de los sitios web españoles analizados cuentan con información bien o muy bien estructurada, mientras que el $47 \%$ la tiene mal o muy mal estructurada. En cuanto a las páginas de titulaciones de otros países, casi el $60 \%$ de las páginas se han clasificado como bien estructuradas. 


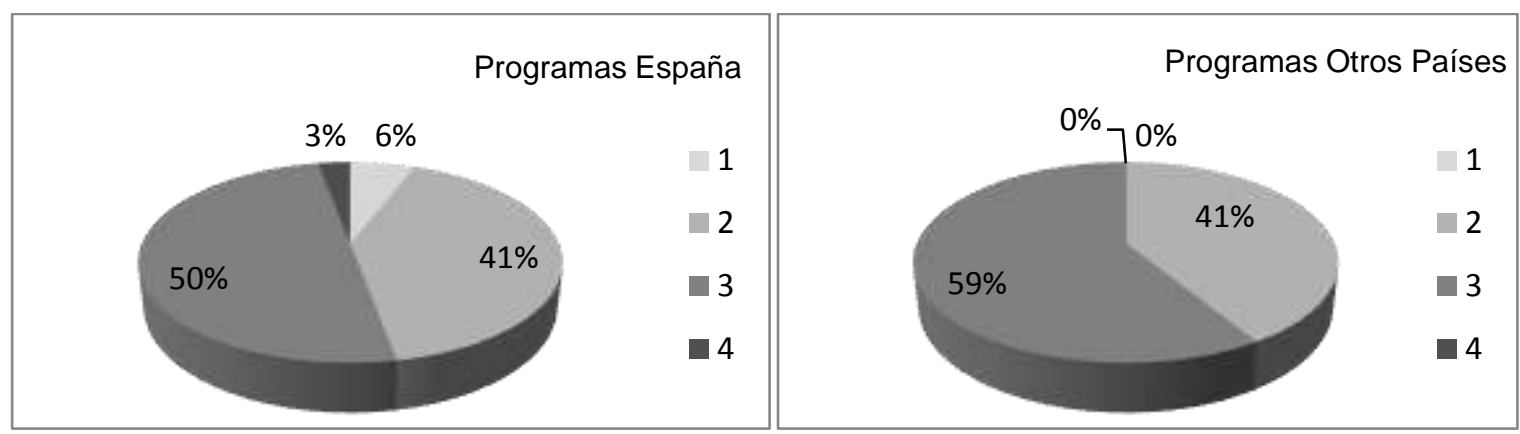

Fig. 2. Porcentaje de webs según la estructuración de su información (1 = "muy mal estructurada", 2 = "mal estructurada" 3 = "bien estructurada" y 4 = "muy bien estructurada)

La accesibilidad es la facilidad para encontrar una página web en buscadores de internet. Sirve de poco contar con una web atractiva y bien diseñada, basada en las directrices actuales del diseño web, si no puede alcanzar buenas posiciones en los principales motores de búsqueda como Google. La figura 3 indica que el $88 \%$ de las webs españolas estudiadas aparecen por debajo de la posición 15\%, un 3\% aparecen entre el puesto $11^{\circ}$ y $15^{\circ}$ y el $9 \%$ restante aparecen entre las primeras 10 webs posicionadas. En el caso de las webs de las titulaciones de otros países, también un porcentaje muy alto $(91 \%)$ aparecen a partir de la $15^{a}$ posición y el $9 \%$ restante aparecen en las 5 primeras posiciones. El porcentaje de webs en las 5 primeras posiciones en las titulaciones de otros países es el triple que en las de las españolas, lo que permite que la información llegue a más futuros doctorandos y a otros agentes implicados en estos estudios.

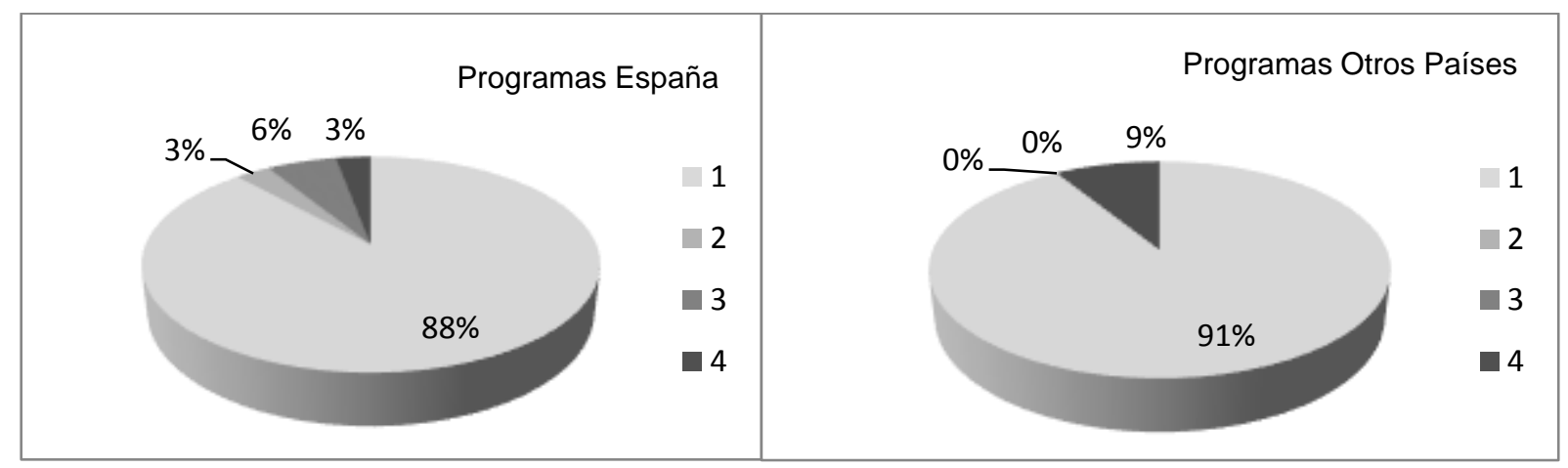

Fig. 3. Porcentaje de webs según la accesibilidad de su información ( 1 = por encima del puesto 15우 2 = entre el puesto $11^{\circ}$ y $15^{\circ} ; 3=$ entre el puesto $6^{\circ}$ y $10^{\circ} ; 4=$ entre el puesto $1^{\circ}$ y $5^{\circ}$ ).

\section{CONCLUSIONES}

La información que aparece en las webs, acerca de los programas de doctorado, todavía es escasa.

Los ítems incluidos en el grupo Accesibilidad son los más frecuentes en las páginas web analizadas, aunque menos en el caso de las titulaciones americanas y asiáticas. Parece que las universidades son conscientes de que es una información muy valiosa para los futuros estudiantes. Los criterios de admisión son el primer paso para que el alumno acceda o descarte matricularse en la titulación y están presentes en al menos el $40 \%$ de las titulaciones.

En cuanto a los Medios disponibles, contenido que complementa al anterior (Accesibilidad), se considera negativa la escasez de esta información tan relevante: número de profesores e información complementaria sobre ellos. El conocimiento de las líneas de investigación de personal del programa resulta relevante para los futuros doctorandos, puesto que la elección del programa estará condicionada a que encuentre entre el profesorado al investigador adecuado para el desarrollo de su trabajo.

La existencia de información relativa a la Difusión, Relevancia y Resultados de investigación es escasa en general. La relativa a la Difusión puede resultar menos relevante, en un principio, para el futuro estudiante. No así para las instituciones, que podrían emplearlo para dar una imagen de calidad investigadora. En el caso de la Relevancia, el estudiante en este tipo de programas valora positivamente la existencia de proyectos de investigación o de alumnos becados, puesto que aportan información sobre oportunidades de financiación para su trabajo. Esta información refleja la calidad de los programas y de sus investigadores, y es un factor muy importante para las instituciones. La Eficacia de los resultados se considera de importancia similar a los anteriores grupos y la cantidad de información sigue siendo escasa. Hay que considerar que 
tanto para los alumnos como para las universidades, el número de tesis terminadas es también un indicador de rendimiento y producción. Solamente las titulaciones españolas y europeas reflejan alguna información sobre la Internacionalización de los programas incluyendo las universidades participantes, que puede ser explicado nuevamente por la pertenencia en ambos casos al EEES. Los programas americanos y asiáticos no incluyen ninguna referencia a los ítems de este grupo. Sería conveniente analizar las causas por las cuales las instituciones de estos continentes no consideran su inclusión en la publicidad de sus programas.

Otro aspecto con escasa o nula información en las webs es la Calidad. La falta de datos sobre indicadores de calidad en la información pública de las instituciones ofrece una mala imagen de las mismas. En España cada vez es más común la implantación de SIGC en todo tipo de instituciones, mostrando procedimientos que pretenden simplificar o facilitar las actividades de la institución. Este dato solo aparece con cierta frecuencia en las titulaciones españolas. Otro aspecto negativo es que no se refleja en las páginas webs la realización de encuestas de satisfacción a ingresados y egresados. El resultado de las encuestas puede ser un atractivo más para el futuro estudiante que considera matricularse en el programa. La presencia de los ítems de este grupo es nula en el caso de las titulaciones americanas y asiáticas. Sería muy conveniente averiguar la razón de que las instituciones no publiquen la información relativa a la calidad.

La existencia o ausencia de determinada información se convierte pues en una potente herramienta de toma de decisiones. La falta de información referente a ítems como, por ejemplo, número de becas, premios de tesis o idiomas de realización del trabajo, puede dar la sensación de que no existen en el programa (de existir, sería negativo para institución no mostrarla). Por tanto, el programa en el que se incluye refuerza su posición respecto al resto. Convendría analizar en profundidad el verdadero motivo por el que falta tanta información, ya que los criterios analizados presentan un gran interés para el desarrollo de un perfil profesional e investigador con mayor proyección internacional.

Al aplicar el test de diferencia de proporciones a los ítems presentes en las páginas de las titulaciones, se obtiene que las titulaciones españolas y las europeas son las que presentan mayores similitudes, posiblemente a causa de que las universidades analizadas son de países del EEES, lo que supone una tendencia a la uniformidad de criterios. En cambio, cuando se trata de comparar con el resto de continentes, las diferencias son mucho mayores, lo que denota que la organización tanto a nivel español como europeo difiere con la del resto de continentes.

Por otro lado, los resultados sobre la calidad de las webs de las universidades indican que, en líneas generales, la mayoría presentan una información clara y estructurada, lo que facilita al futuro estudiante encontrar de manera rápida, sencilla e intuitiva los datos relevantes del programa. Sin embargo, los resultados son menos positivos en lo referente a los diseños, puesto que las páginas webs son, en general, poco atractivas a causa de su excesiva sencillez y escaso dinamismo. Respecto al posicionamiento web, se puede observar que es, en general, mejorable. Teniendo en cuenta que las búsquedas de información se realizan a través de grandes buscadores como Google, sería positivo para la publicidad de las instituciones realizar un pequeño esfuerzo y posicionarse mejor en los resultados que consideren adecuados para cada titulación. De esta manera, el futuro estudiante estará más cerca de decidirse por la titulación.

Este trabajo puede ser, por tanto, un excelente punto de partida para poder mejorar las carencias de información, o bien para focalizar los esfuerzos de los programas de doctorado en incidir en los contenidos menos frecuentes con objeto de alcanzar mejores parámetros de calidad, lo que podría aumentar del interés de los alumnos en cursar un doctorado.

\section{REFERENCIAS}

Aguado, P. y otros 16 autores, Analysis of the information about doctoral degrees presented in the Spanish universities websites. Proceedings of the $15^{\text {th }}$ WMSCI 2011, 391-394 (2011). (en línea: http://oa.upm.es/12420/2/INVE_MEM_2011_104726.pdf, acceso: 25-3-2015)

Al-Debei, M. M. The quality and acceptance of websites: an empirical investigation in the context of higher education. International Journal of Business Information Systems, 15(2), 170-188 (2014)

Altbach, P.G. y J. Knight, The Internationalization of Higher Education: Motivations and Realities. Journal of Studies in International Education, 11(3/4): 290-305 (2007)

Ayuga-Téllez, E., La Información en Red Relativa a los Títulos de Doctor de la Universidad Española. Memoria del proyecto del Programa de Estudios y Análisis del Ministerio de Educación y Ciencia (2010) (en línea: http://138.4.83.162/mec/ayudas/CasaVer.asp?P=29 384, acceso: 29-5-2015) 
Ayuso García, M.D. y V. Martínez Navarro, Protocolo de evaluación de fuentes y recursos informativos en la sociedad del conocimiento: propuestas, enfoques y tendencias. Revista General de Información y Documentación, 15(1), 21-53 (2005)

Aznar Minguet, P.; UII, M.A.; Piñero, A. y Martínez Agut, M.P., La sostenibilidad en la formación universitaria: Desafíos y oportunidades. Educación XX1, 17 (1), 131-158 (2014)

Benito, M. y R. Romera, Modeling the enrollment demand of masters programs for the Spanish public university system. Scientometrics, 91(1), 113-130 (2012)

Buela-Casal, G., y Á. Castro, Análisis de la evolución de los programas de doctorado con Mención de Calidad en las universidades españolas y pautas para su mejora. Revista de Investigación en Educación, 5, 49-60 (2009)

Cardoso, E.O; Cerecedo, M.T y Vanegas, E.A. Las Competencias Docentes en los Programas de Posgrado en Administración: Un Estudio de Diagnóstico. Formación. Universitaria, 6(2), 43-50 (2013).

de Miguel Díaz, F. M. Evaluación y mejora de los estudios de doctorado. Revista de educación, (352): 569581 (2010).

Dávila, M. Tendencias internacionales en posgrados. Integración y Conocimiento, (1): 18-26 (2012)

Eurostat,. Tertiary education participation (2014). (en línea: http://appsso.eurostat.ec.europa.eu/nui/show.do, acceso: 23-11-2015)

Fornas Carrasco, R. Criterios para evaluar la calidad y la fiabilidad de los conocimientos en Internet. Revista española de documentación científica, 26(1), 75-80 (2003)

Gijón Puertas, J., y E. Crisol Moya, La internacionalización de la Educación Superior: El caso del Espacio Europeo de Educación Superior. REDU: Revista de Docencia Universitaria, 10(1), 389-414 (2012)

González-Riaño, M., R. Repiso, y E. Delgado López-Cózar. Repercusión de los rankings universitarios en la prensa española. Revista Española de Documentación Científica 37(3) : e055. (2014)

Hernandez, C.A; Tavera, M.E y Jimenez, M. Seguimiento de Egresados en Tres Programas de Maestría en una Escuela del Instituto Politécnico Nacional en México. Formación. Universitaria, 5(2), 41-52 (2012)

Horgué Baena , C. La ordenación del Doctorado. Revista de Administración Pública, 189, septiembrediciembre, 365-401 (2012)

Krüger, K. y A. Molas,. Rankings mundiales de universidades: objetivos y calidad. Ar@cne. Revista Eléctronica de Recursos en Internet sobre Geografía y Ciencias Sociales. (en línea: http://www.ub.edu/geocrit/aracne/aracne-129.htm acceso:10-01-2016), 129, 1 de enero de 2010 (2010)

MECD, Datos básicos del sistema universitario español. Curso 2014-2015. Ministerio de Educación, Cultura y Deporte (MECD). 176 pp (2015). (en línea: http://www.mecd.gob.es/dms/mecd/educacion-mecd/areaseducacion/universidades/estadisticas-informes/datos-cifras/Datos-y-Cifras-del-SUE-Curso-2014-2015.pdf, acceso: 15-12-2015)

Moguerou, P., Doctoral and postdoctoral education in science and engineering: Europe in the international competition. European Journal of Education, 40(4), 367-392 (2005)

Noguero, F. L. El análisis de contenido como método de investigación. XXI. Revista de educación, (4), 167180 (2002)

Pinto, M., D. Guerrero Quesada y X. Granell, Dissemination of information and visibility of the European Higher Education Area through the websites of Spanish universities: a longitudinal metric analysis, 20072012. Scientometrics, 98(2), 1235-1255 (2014).

Robins, D., y J. Holmes, Aesthetics and credibility in web site design. Information Processing \& Management, 44(1), 386-399 (2008)

Gobierno de España, La Estrategia Universidad 2015 (EU 2015). (2008) (en línea: http://www.mecd.gob.es/eu2015, acceso: 25-3-2015)

Sursock, A. y H. Smidt,. Trends 2010: a decade of change in European Higher Education. EUA Publications (2010). (en línea: http://www.eua.be/publications/eua-reports-studies-and-occasional-papers.aspx, acceso: 29-5-2015)

Wainerman, C. y C. Tuñón, La eficiencia de los programas doctorales y su evaluación. Revista Argentina de educación Superior, Año 5, 6, 167-188 (2013)

Webometrics (2015). (en línea: http://www.webometrics.info, acceso:10-10-2015) 